\title{
The diagnostic performance of urinary free cortisol is better than the cortisol:cortisone ratio in detecting de novo Cushing's syndrome: the use of a LC-MS/MS method in routine clinical practice
}

\author{
Filippo Ceccato, Giorgia Antonelli' ${ }^{1}$ Mattia Barbot, Marialuisa Zilio, Linda Mazzai, \\ Rosalba Gatti ${ }^{2}$, Martina Zaninotto ${ }^{3}$, Franco Mantero, Marco Boscaro, \\ Mario Plebani ${ }^{1,3}$ and Carla Scaroni \\ Endocrinology Unit, Department of Medicine DIMED, University-Hospital of Padova, via Ospedale Civile 105, \\ 35128 Padova, Italy, 'Laboratory Medicine Unit, Department of Medicine DIMED, University-Hospital of Padova, \\ via Giustiniani 2, 35128, Padova, Italy, ${ }^{2}$ Endocrinology Unit, University-Hospital of Padova, via Ospedale Civile 105, \\ 35128 Padova, Italy and ${ }^{3}$ Department of Laboratory Medicine,University-Hospital of Padova, via Giustiniani 2, \\ 35128 Padova, Italy
}

\author{
Correspondence \\ should be addressed \\ to C Scaroni \\ Email \\ carla.scaroni@unipd.it
}

\begin{abstract}
Objective: The Endocrine Society Clinical Guidelines recommend measuring 24-h urinary free cortisol (UFF) levels using a highly accurate method as one of the first-line screening tests for the diagnosis of Cushing's Syndrome (CS). We evaluated the performance of UFF, urinary free cortisone (UFE), and the UFF:UFE ratio, measured using a liquid chromatography-tandem mass spectrometry (LC-MS/MS) method.

Subjects and methods: The LC-MS/MS was used to analyze UFF and UFE levels in 43 surgically confirmed CS patients: 26 with Cushing's disease (CD, 16 de novo and ten recurrences), 11 with adrenal CS and six with ectopic CS; 22 CD patients in remission; 14 eu-cortisolemic CD patients receiving medical therapy; 60 non-CS patients; and 70 healthy controls. Sensitivity and specificity were determined in the combined groups of non-CS patients, healthy controls, and CD in remission.

Results: UFF $>170 \mathrm{nmol} / 24 \mathrm{~h}$ showed $98.7 \%$ specificity and $100 \%$ sensitivity for de novo CS, while sensitivity was $80 \%$ for recurrent CD patients, who were characterized by lower UFF levels. The UFF:UFE and UFF+ UFE showed lower sensitivity and specificity than UFF. Ectopic CS patients had the highest UFF and UFF:UFE levels, which were normal in the CD remission patients and in those receiving medical therapy.

Conclusions: Our data suggest high diagnostic performance of UFF excretion measured using LC-MS/MS, in detecting de novo CS. UFF:UFE and UFF + UFE assessments are not useful in the first step of CS diagnosis, although high levels were found to be indicative of ectopic CS.

\section{Introduction}

Cushing's syndrome (CS) is a rare disease with patients often presenting with a less than clear clinical picture and at times only isolated signs, although many of its typical symptoms are common in the general population $(1,2)$.
When efficient diagnostic and screening strategies are used in specific populations (diabetes mellitus and osteoporosis), a significantly higher number of CS diagnoses are made $(3,4)$. (c) 2014 European Society of Endocrinology Printed in Great Britain
Published by Bioscientifica Ltd. 
The Endocrine Society Clinical Practice Guidelines (1) recommends initially testing for CS using one test with high diagnostic accuracy: 24-h urinary free cortisol (UFF) excretion, late night salivary cortisol (LNSC), or $1 \mathrm{mg}$ dexamethasone suppression test (DST). It has been seen that many of the commercially available antibody-based immunoassays for measurement of UFF levels can give falsely high cortisol values due to cross-reactivity with its metabolites (5). While liquid chromatography-tandem mass spectrometry (LC-MS/MS) is considered an accurate method to determine UFF levels (1), it is not widely used in clinical practice $(5,6,7)$.

The balance between cortisol and cortisone (E) levels is predominantly regulated by $11-\beta$-hydroxysteroid dehydrogenase (11- $\beta$-HSD) types 1 and $2(5,8)$. In the event of excessive cortisol secretion, as occurs in CS, 11- $\beta$ HSD2 can be saturated, causing an increase in the UFF:UFE, which has been described in ectopic CS (9); the $\mathrm{UFF}+\mathrm{UFE}$ sum is not, instead, influenced by 11- $\beta$-HSD 2.

The aim of this study was to determine the accuracy of UFF levels, measured using LC-MS/MS in distinguishing CS from non-CS, and to examine the additional diagnostic value of the UFF:UFE ratio for making a differential diagnosis of CS.

\section{Subjects and methods}

\section{Patients}

Two hundred and nine patients referred to the Endocrinology Unit of the University of Padova Medical Center between December 2011 and December 2013 were assessed. These included:

i) Forty-three patients with CS (age at diagnosis $48 \pm 18$ years). CS suspicion was based on the lack of cortisol suppression $<50 \mathrm{nmol} / \mathrm{l}$ after $1 \mathrm{mg}$ DST and LNSC levels $>5.24 \mathrm{ng} / \mathrm{ml}$ (10) in at least two samples. All the subjects had final histological confirmation of CS: 26 had Cushing's disease (CD, 16 de novo and ten recurrences after a minimum of 12 months of remission), 11 with adrenal CS (six adenoma and one carcinoma), and six with ectopic CS (two pancreatic and four bronchial neuroendocrine tumors with positive adrenocorticotropic hormone (ACTH) immunostaining). The following parameters were assessed in each of these patients: body weight $(\mathrm{kg})$, waist circumference (normal: $<94 \mathrm{~cm}$ in males and $<80 \mathrm{~cm}$ in females), BMI $\left(\mathrm{kg} / \mathrm{m}^{2}\right)$, potassium levels (normal range $3.4-4.5 \mathrm{mEq} / \mathrm{l}$ ), blood pressure (hypertension was defined as systolic blood pressure $\geq 130 \mathrm{mmHg}$ and/or diastolic $\geq 85 \mathrm{mmHg}$ or on antihypertensive treatment), lipid profile (dyslipidemia was diagnosed when plasma triglycerides $\geq 1.7 \mathrm{mmol} / 1$ or $\mathrm{HDL}<1 \mathrm{mmol} / 1$ in males and $<1.3 \mathrm{mmol} / 1$ in females, or in patients receiving treatment), diabetic mellitus (fasting glucose $\geq 5.5 \mathrm{mmol} / \mathrm{l}$ or patients being treated for diabetes). Patients with three or more of the following parameters were considered as being affected with the metabolic syndrome: elevated waist circumference, increased triglycerides, reduced HDL levels, hypertension, and diabetic mellitus (11).

ii) Twenty-two patients (age $50 \pm 15$ years) in remission from CD (median follow-up 52 months, range 14-96). Patients were considered in remission after a minimum of 6 months following pituitary surgery and when at least two of the following criteria were met: i) need for postoperative corticosteroid replacement therapy; ii) low serum cortisol levels early after surgery ( $<50 \mathrm{nmol} / \mathrm{l}$, low/normal morning serum cortisol level for at least 6 months after pituitary surgery; and iii) significant changes in clinical features (12). None of the patients received glucocorticoid substitutive therapy for at least 1 month before urine collection; all had normal LNSC and cortisol levels $<50 \mathrm{nmol} / \mathrm{l}$ after $1 \mathrm{mg}$ DST (both were assessed every 12 months during the follow-up).

iii) Fourteen $\mathrm{CD}$ patients (age $52 \pm 14$ years) receiving medical therapy and showing normal LNSC levels and cortisol suppression $<50 \mathrm{nmol} / \mathrm{l}$ after $1 \mathrm{mg}$-DST: eight patients were being treated with ketoconazole (two with $200 \mathrm{mg} /$ daily, four with $400 \mathrm{mg} /$ daily, and two with $600 \mathrm{mg} /$ daily, duration of therapy 16-54 months), six patients were receiving both ketoconazole (400-600 mg/daily) as well as cabergoline (3.5 mg/weekly, duration of therapy 16-54 months), five were receiving pasireotide $(600 \mu \mathrm{g}$ b.i.d., for 10 months) and one temozolamide (400 mg daily for 5 days every 28 days, the patient underwent 18 cycles of therapy).

iv) Sixty subjects with medical conditions suggestive of hypercortisolism (age $45 \pm 16$ years), cortisol $<50 \mathrm{nmol} / \mathrm{l}$ after $1 \mathrm{mg}$ DST, and normal LNSC (at baseline and after 12 months).

v) Seventy healthy subjects (age $47 \pm 21$ years) recruited from the staff of the Endocrinology Unit and the Laboratory Medicine Department of the University of Padova Medical Centre. All underwent a comprehensive clinical examination and detailed medical 
histories were taken. None showed signs or symptoms of hypercortisolism, none had a history of adrenal incidentaloma or severe and/or chronic illness (in particular of an endocrine origin). None were taking exogenous glucocorticoids or drugs that could interfere with the hypothalamic-pituitary-adrenal axis.

The study was carried out in accordance with the guidelines in the Declaration of Helsinki, the Local Ethics Committee approved the protocol, and all patients/ subjects gave informed consent.

\section{Urinary glucocorticoid analysis}

For 24-h urine collections, the patients were instructed to discard the first morning urine void and to collect all urine for the next $24 \mathrm{~h}$, so that the morning urine void on the second day was the final collection. The sample was kept refrigerated from collection time until it was analyzed: a $10 \mathrm{ml}$ aliquot sample was taken and centrifuged at $3000 \mathrm{~g}$ for $10 \mathrm{~min}$ at room temperature. Then $2 \mu \mathrm{l}$ formic acid and $50 \mu \mathrm{l}$ deuterated internal standard solution $\left(\mathrm{d}_{4}\right.$-cortisol and $\mathrm{d}_{7}$-cortisone) were added to $500 \mu \mathrm{l}$ of urine supernatant or calibrators. The solution was vortexed for $30 \mathrm{~s}$ and centrifuged at $16000 \boldsymbol{g}$ for $5 \mathrm{~min}$ at room temperature. This was followed by $20 \mu$ supernatant added to $200 \mu \mathrm{l} 0.1 \%$ formic acid water solution and placed in the autosampler of the LC-MS/MS. UFF and UFE levels were measured, as is routine in our center, using an Agilent HPLC series 1200 triple quadrupole mass spectrometer, Agilent 6430, equipped with an electrospray ionization source in a positive ionization mode (Agilent Technologies, Palo Alto, CA, USA). The on-line cleanup/enrichment was carried out using a cartridge Zorbax Extend-C18 $2.1 \times 12.5 \mathrm{~mm}, 5 \mu \mathrm{m}$ particle size, and the HPLC separation by a $4.6 \times 50 \mathrm{~mm}, 1.8 \mu \mathrm{m}$ particle size, analytical column Zorbax Eclipse XDB-C18 (Agilent Technologies). Quantitative analysis was carried out in the multiple reaction monitoring mode. The method was linear up to 625 and $1125 \mathrm{nmol} / \mathrm{l}$, with a lower quantification limit of 5 and $6 \mathrm{nmol} / 1$ for UFF and UFE respectively. Within-run and between-run coefficients of variation (CV) were $<5$ and $6 \%$ for UFF and 6 and $8 \%$ for UFE respectively. The mean recoveries were $106 \%$ for UFF and $104 \%$ for UFE. The intra-individual variability of UFF was determined by calculating the $\mathrm{CV}$ $(\mathrm{CV}=$ s.D./mean $\times 100)$.

All the patients provided two to five complete 24-h urine collections, the average values from all of the collections were used in the final analysis. The healthy controls provided one sample.

The reference range used in our department, determined using the LC-MS/MS method described above, is for UFF 16-170 nmol/24 h, UFE 41-364 nmol/24 h, and UFF:UFE 0.14-1.09 (13); these values are comparable with others obtained using LC-MS/MS methods $(5,6,14)$.

\section{Data and statistical analysis}

UFF, UFE, UFF:UFE, and UFF+UFE excretion in the different CS groups was compared. The de novo CS group was defined as: de novo $\mathrm{CD}+$ adrenal $\mathrm{CS}+$ ectopic CS. The rate of false-positive test results in the combined groups of non-CS patients, healthy controls, and CD remission patients (considering the last as a group in which recurrence was possible) was determined. Continuous data are shown as mean and s.D. or median and range. We compared the groups using the Mann-Whitney $U$-test for non-parametrical data; categorical variables were compared using the $\chi^{2}$ test or Fisher's exact test when the cell count was $<5$. Sensitivity and specificity were calculated at different cut-off levels to carry out receiver operating curve (ROC) analyses. Linear regression was used to examine the relationship between UFF or UFF:UFE and age. Statistical analysis was performed using the SPSS 17 Software package (SPSS, Inc.). The significance level was set at $P$ value $<0.05$ for all the tests.

\section{Results}

\section{UFF excretion}

UFF levels were higher in the CS patients with respect to all the other groups, and the highest values were found in the ectopic CS patients and in the single patient with adrenal carcinoma (Table 1 and Fig. 1). The CV ranged between 4 and 114\% (median 39\%).

Sensitivity and specificity of UFF $>170 \mathrm{nmol} / 24 \mathrm{~h}$ were respectively 95.3 and $98.7 \%$ for the CS patients (Table 2), with positive and negative predictive values of 95.3 and $98.5 \%$. The best calculated cut-off value according to ROC-curve analysis was $197 \mathrm{nmol} / 24 \mathrm{~h}$ (sensitivity $95.3 \%$ and specificity 100\%). When only the 33 de novo $\mathrm{CS}$ patients were considered, the sensitivity and specificity of UFF $>170 \mathrm{nmol} / 24 \mathrm{~h}$ were 100 and $98.7 \%$ respectively with positive and negative predictive values 94.3 and 100\% respectively; according to ROC analyses, UFF $>197 \mathrm{nmol} / 24 \mathrm{~h}$ showed 100\% sensitivity and specificity for de novo CS. Two of the ten recurrent CD 
Table 1 UFF, UFE, and UFF:UFE median levels (range) in nmol/24 $\mathrm{h}$ in selected groups of patients.

\begin{tabular}{|c|c|}
\hline Group & \\
\hline $\operatorname{CS}(n=43)$ & $\begin{array}{l}\text { CD }(n=26) \\
\text { CD de novo }(n=16) \\
\text { CD recurrence }(n=10) \\
\text { Adrenal CS }(n=11) \\
\text { Ectopic CS }(n=6)\end{array}$ \\
\hline CS de novo $(n=33)$ & $\begin{array}{l}\text { CD de novo }(n=16) \\
\text { Adrenal CS }(n=11) \\
\text { Ectopic CS }(n=6) \\
\text { CD on medical therapy } \\
\quad(n=14)\end{array}$ \\
\hline Controls $(n=152)$ & $\begin{array}{l}\text { CD in remission }(n=22) \\
\text { Non-CS }(n=60) \\
\text { Healthy controls } \\
\quad(n=70)\end{array}$ \\
\hline
\end{tabular}

\begin{tabular}{c}
\hline $\mathbf{M} / \mathbf{F}$ \\
\hline $9 / 17$ \\
$7 / 9$ \\
$2 / 8$ \\
$1 / 10$ \\
$1 / 5$ \\
$9 / 24$ \\
\\
$4 / 10$ \\
$5 / 17$ \\
$12 / 48$ \\
$20 / 50$ \\
\hline
\end{tabular}

\begin{tabular}{c}
\hline UFF \\
\hline $398(27-3344)^{a, b, c, d}$ \\
$548(281-3344)^{a, b, c, d, e}$ \\
$289(27-948)^{a, b, c, d}$ \\
$808(208-10360)^{a, b}$ \\
$5992(421-29120)^{a, b, f}$ \\
$808(208-29120)^{a, b}$
\end{tabular}

$64(27-168)$

$58(15-151)$

$51(10-180)$

$58(16-186)$

\begin{tabular}{l}
\hline UFE \\
\hline $448(58-1606)^{a, b, c, d}$ \\
$576(286-1606)^{a, b, c, d, e}$ \\
$306(58-659)^{a, b, c, d}$ \\
$693(192-1325)^{a, b}$ \\
$884(365-1840)^{a, b, f}$ \\
$619(192-1840)^{a, b}$
\end{tabular}

$148(13-464)$

$151(19-375)$

$131(26-426)$

$155(36-463)$

\begin{tabular}{l}
\hline UFF:UFE \\
\hline $1.05(0.45-2.26)^{a, b, c, d}$ \\
$1.23(0.47-2.26)^{a, b, c, d}$ \\
$1.00(0.45-1.91)^{a, b, c, d}$ \\
$1.80(0.70-12.95)^{a, b}$ \\
$4.92(1.15-26.47)^{a, b, f}$ \\
$1.52(0.47-26.47)^{a, b}$
\end{tabular}

$0.33(0.20-0.62)$

$0.41(0.28-0.86)$

$0.41(0.16-1.66)$

$0.38(0.14-1.19)$

$\mathrm{M}$, male; F, female; UFF, urinary free cortisol; UFE, urinary free cortisone.

${ }^{\mathrm{a}} P<0.001$ vs healthy subjects.

${ }^{\mathrm{b}} P<0.001$ vs non-CS.

${ }^{C} P<0.001$ vs $C D$ in medical therapy.

${ }^{\mathrm{d}} P<0.001$ vs $\mathrm{CD}$ in remission.

${ }^{\mathrm{e}} P<0.05$ vs $C D$ recurrence.

${ }^{\mathrm{f}} P<0.001$ vs $\mathrm{CD}$.

patients had normal UFF levels, resulting in an $80 \%$ sensitivity. There were no false-negative UFF results in the patients with adrenal or ectopic CS.

Those CS patients with UFF $<1.5$ times the upper normal limit $(<255 \mathrm{nmol} / \mathrm{l})$ were considered as being affected with mild hypercortisolism. None of the ectopic CS patients did, but one out of 11 of the adrenal CS (9\%) and four out of $26(15 \%)$ of the CD patients had a mild form and all were with recurrent CD. While UFF levels were higher in the de novo than in the recurrent $\mathrm{CD}$ patients $(P<0.05$, Table 1$)$, the frequency of mild hypercorticism was higher in the latter (four out of ten, $40 \%$ ) with respect to the former ( 0 out of $16, P<0.05$ ).

\section{UFF:UFE ratio}

The diagnostic performance of UFE was worse than that of UFF (Table 2); the ROC-curve based cut-off $(281 \mathrm{nmol} / 24 \mathrm{~h})$ showed a higher accuracy in the detection of CS with $86 \%$ sensitivity and $93.4 \%$ specificity. Seven out of ten of the recurrent CD patients had a normal UFE excretion, while all of the ectopic CS patients had increased levels.

The optimal cut-off for UFF:UFE according to ROCcurve analysis was 0.73 (sensitivity $86 \%$ and specificity $92.8 \%$ ). When a threshold of 1.09 (upper reference limit (15)) was used, the UFF:UFE ratio showed a lower degree
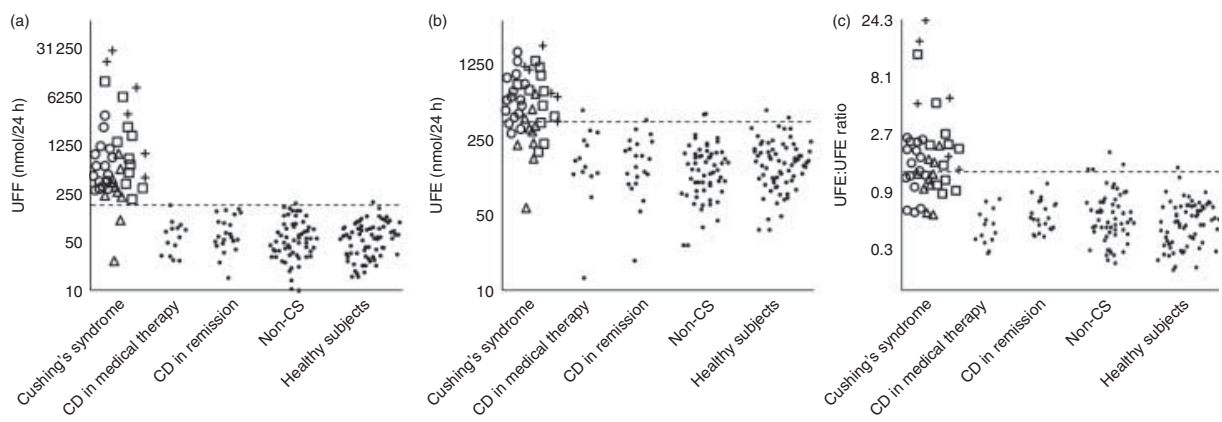

\section{Figure 1}

Scatter plot of UFF (a), UFE (b), and UFF:UFE (c) in the different populations: Cushing's syndrome $(n=43), C D$ receiving medical therapy $(n=14), C D$ in remission $(n=22)$, non-CS $(n=60)$, and healthy controls $(n=70)$. Full black line indicates ROC-calculated threshold values in the de novo CS and non-CS patients. Open circle, de novo $\mathrm{CD}(n=16)$; open triangle, recurrent $\mathrm{CD}(n=10)$; open square, adrenal-CS $(n=11)$; and cross, ectopic CS $(n=6)$; filled circle, control groups described above. 
Table 2 Sensitivity (SE, \%), specificity (SP, \%), and AUC for UFF, UFE, and UFF:UFE.

\begin{tabular}{|c|c|c|c|c|c|c|}
\hline & \multicolumn{3}{|c|}{ CS } & \multicolumn{3}{|c|}{ de novo CS } \\
\hline & AUC & SE & SP & AUC & SE & SP \\
\hline UFF $>170 \mathrm{nmol} / 24 \mathrm{~h}$ & 0.976 & 95.3 & 98.7 & 1.00 & 100.0 & 98.7 \\
\hline UFE > $364 \mathrm{nmol} / 24 \mathrm{~h}$ & 0.941 & 69.8 & 96.7 & 0.979 & 81.8 & 96.7 \\
\hline UFF:UFE > $>1.09$ & 0.940 & 58.1 & 96.7 & 0.953 & 66.7 & 96.7 \\
\hline
\end{tabular}

AUC, area under the curve.

of accuracy in the detection of CS (Table 2). Ectopic CS patients had the highest UFF:UFE values; the ROC generated threshold of UFF:UFE >1.51 showed $83.3 \%$ sensitivity and $73.1 \%$ specificity (AUC 0.885 ) in distinguishing ectopic from pituitary ACTH secretion. The UFF:UFE ratio was normal in the two recurrent $\mathrm{CD}$ patients who had normal UFF and in all of the eu-cortisolemic CD patients (Table 1). The diagnostic accuracy of the UFF + UFE sum was similar to that of the UFF:UFE ratio. It was higher in the CS with respect to the non-CS patients and the healthy controls, it was higher in the ectopic CS with respect to the $\mathrm{CD}$ patients, and it showed higher sensitivity (93\%) and specificity (94.7\%) in detection of CS (UFF + UFE $>432 \mathrm{nmol} / 24 \mathrm{~h}$, AUC 0.971). It had only a slightly lower diagnostic accuracy in distinguishing between ectopic CS and CD (sensitivity $83.3 \%$ and specificity $69.2 \%$ if UFF+UFE > $1264 \mathrm{nmol} / 24 \mathrm{~h}$, AUC 0.833).

The patients with an UFF:UFE $>1.09$ showed higher UFF levels than did those with the ratio $<1.09$ (for CS median UFF 1115 vs $308 \mathrm{nmol} / 24 \mathrm{~h}, P<0.001$ and for CD median 630 vs $305 \mathrm{nmol} / 24 \mathrm{~h}, P<0.01$ ); there were no differences in these groups with regard to body weight, BMI, prevalence of diabetes, hypertension, dyslipidemia, or metabolic syndrome. CS patients with UFF:UFE $>1.09$ had lower waist circumference (median 94 vs $108 \mathrm{~cm}$, $P<0.01$ ) and lower potassium levels (median 3.7 vs $3.9 \mathrm{mEq} / \mathrm{l}, P<0.05$ ) as well as a higher rate of hypokalemia. Seven, in fact, out of 25 (one CD, one adrenal, and five ectopic CS) patients with UFF:UFE $>1.09$ had serum potassium levels $<3.4 \mathrm{mEq} / 1$ (median 2.4 , range $1.8-2.8 \mathrm{mEq} / \mathrm{l}$ ) and a median UFF:UFE of 5.21 (range 1.15-26.47), while none of the patients with UFF:UFE ratio $<1.09$ had hypokalemia $(P<0.05)$. UFF:UFE levels were normal (in seven out of ten) or only slightly elevated in the recurrent $\mathrm{CD}$ patients.

\section{Sex and age difference}

No gender differences linked with UFF or UFF:UFE values were found in any of the groups. Considering those patients with ectopic CS or adrenal carcinoma as malignant CS, UFF and UFF:UFE levels decreased with age in the nonmalignant CS (UFF $y=-32.958 x+2347.5, r^{2}=0.2373$, $P<0.01$ and UFF:UFE $y=-0.0175 x+2.1395, r^{2}=0.1341$, $P<0.05)$. In the de novo non-malignant CS patients (to avoid bias linked with lower UFF levels in recurrent CD) UFF and UFF:UFE levels decreased with age: UFF $y=-44.059 x+3111.2, r^{2}=0.3672, P<0.01$ and UFF:UFE $y=-0.0247 x+2.6218, r^{2}=0.2423, P<0.05$; (Fig. 2).

UFF levels decreased with age $(y=-1.2243 x+135.38$, $\left.r^{2}=0.2071, P<0.05\right)$ to a greater degree in the CD remission patients with respect to the healthy subjects $\left(r^{2}=0.108, P<0.01\right)$; no age-related variation was found in UFF or in the UFF:UFE ratio in the CD patients receiving medical therapy or in the non-CS subjects.

\section{Discussion}

When evaluating patients for the diagnosis of CS, the Endocrine Society recommends determination of UFF levels

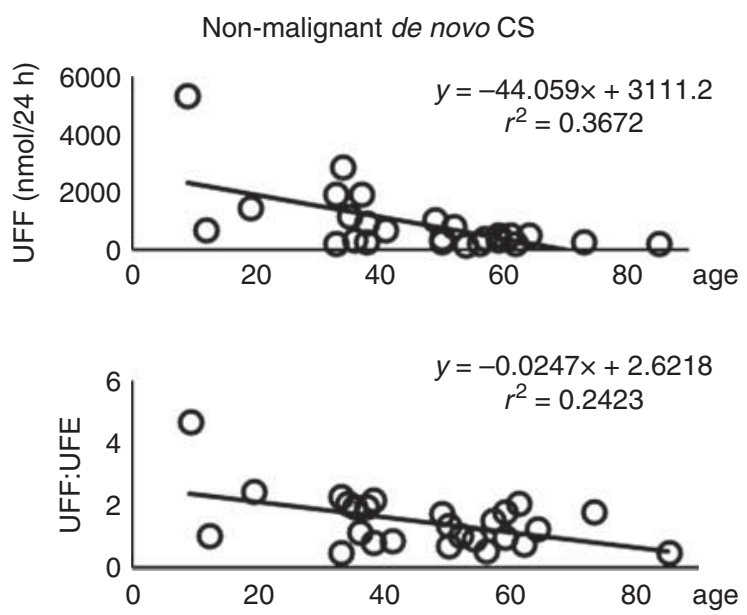

Figure 2

Logistic regression in age-related decreasing UFF levels and UFF:UFE ratio in the non-malignant de novo Cushing's syndrome patients. Open circles represent patient's measurements. 
using highly accurate methods instead of commercially available antibody-based immunoassays $(1,5,15)$. In this study, UFF levels, routinely determined in our centre using the LC-MS/MS method, were compared among healthy subjects, CS patients, and non-CS patients presenting clinical features suggestive of hypercortisolism but with normal LNSC and cortisol $<50 \mathrm{nmol} / \mathrm{l}$ after $1 \mathrm{mg}$ DST. The diagnostic value of the UFF:UFE ratio was also assessed $(5,9,16)$.

This is the first work, to our knowledge, assessing healthy controls and a series of consecutive surgically confirmed CS patients as well as patients in whom the diagnosis of CS was excluded, with the intent of analyzing the sensitivity and specificity of UFF values measured using the LC-MS/MS method.

UFF values obtained using the LC-MS/MS method did not produce any false-negative test results in the de novo CS patients, without mild hypercortisolism: the reference ranges for UFF used in a laboratory are of utmost importance in view of their crucial role in cases of suspected hypercortisolism. In accordance with (17) report, we found, moreover, a high intrapatient variability in two or more samples collected for evaluation of UFF levels.

In our center, a patient is considered as having recurrent $\mathrm{CD}$ when, after a stable clinical/biochemical remission, he/she shows signs of worsening health status and hypercortisolism confirmed by elevated LNSC levels and lack of cortisol suppression to $1 \mathrm{mg}$ DST (we routinely used the most sensitive cut-off even in this case). Confirming previously described data regarding LNSC $(10,18,19)$, higher UFF excretion was found in the de novo with respect to the recurrent $C D$ patients, and a higher rate of mild hypercortisolism was found in the latter. We also found normal UFF excretion in two out of ten recurrent CD patients. One study in the literature, in fact, reported that elevated UFF levels appear later with respect to other tests in recurrent CD patients (20). During the routine follow-up of these patients, early diagnosis of recurrence (based on pathological results of two other screening tests) can be made even in the event that UFF levels are normal or only slightly elevated, due to lower sensitivity and specificity of UFF in this particular clinical setting. It is, nevertheless, important to recognize recurrence at an early stage when hypercortisolism is still mild. Endocrinologists should thus carry out at least two screening tests rather than two urine collections during the post-operative follow-up of patients who have surgical remission of CD.

UFF:UFE and UFF+UFE have no additional value with respect to UFF in cases of recurrent CD. In accordance with (16) data, we found no differences in the UFF:UFE levels in the healthy controls and CD patients receiving medical therapy.

Just as was reported with regard to LNSC levels, ectopic CS patients had higher UFF, UFF+UFE sum, and UFF:UFE ratios than did the CD patients (10). The UFF:UFE ratio has been reported to be a marker of this type of CS (8) and a higher UFF:UFE ratio was indeed found in the ectopic CS patients studied here. Further studies are needed to establish whether the ratio can be considered as a biomarker for ectopic ACTH, which is of occult origin in $20 \%$ of ACTH-dependent cases (21).

Higher UFF:UFE ratios in CS patients suggest that there is $11-\beta$-HSD2 saturation, which is typical of CS $(5,8,16)$. If we divide our CS into two groups whose UFF:UFE ratios are higher or lower with respect to the local upper reference limit of 1.09 , the group with the higher ratio showed elevated UFF levels. It is probable that only high cortisol levels are able to saturate 11- $\beta$-HSD2 activity leading to an excess of cortisol production that could explain the typical co-morbidities in CS. In addition, the patients with UFF:UFE ratio $>1.09$ had low potassium levels and a high rate of hypokalemia, confirming that excess cortisol saturates 11- $\beta$-HSD2 and leads to cortisolrelated mineralocorticoid activity.

We observed a linear regression in age-related decreasing levels of UFF and UFF:UFE in the non-malignant de novo $\mathrm{CS}$, a finding which needs to be examined by future studies assessing large CS populations (Fig. 2).

This study has some limitations. Although ours is a third-level referral centre for CS, the number of patients studied was relatively low. Stringent inclusion criteria (i.e. the histological confirmation of CS or both normal LNSC and the lower cut-off after $1 \mathrm{mg}$ DST to define non-CS) were, nevertheless, used to enable us to accurately analyze the sensitivity and specificity of UFF. Studies using larger numbers of patients with discordant test results are warranted to verify the diagnostic accuracy of UFF measured using the LC-MS/MS method and to establish the UFF:UFE ratio as a biomarker of ectopic CS.

In conclusion, in view of its accuracy, UFF levels measured using the LC-MS/MS method can be considered a useful element during the routine clinical assessment of patients with suspected hypercortisolism. Measurement of UFF levels was found to be reliable in detection of de novo $\mathrm{CS}$. Its sensitivity in diagnosing recurrent $\mathrm{CD}$ was instead limited, probably due to higher levels of mild hypercortisolism rather than to methodological issues. The UFF:UFE ratio does not appear to be useful in the first step of CS diagnosis, but it may be important in forming the differential diagnosis of ACTH-dependent hypercortisolism. 


\section{Declaration of interest}

The authors declare that there is no conflict of interest that could be perceived as prejudicing the impartiality of the research reported.

Funding

This study did not receive any specific grant from any funding agency in the public, commercial or not-for-profit sector.

\section{References}

1 Nieman LK, Biller BM, Findling JW, Newell-Price J, Savage MO, Stewart PM \& Montori VM. The diagnosis of Cushing's syndrome: an Endocrine Society Clinical Practice Guideline. Journal of Clinical Endocrinology and Metabolism 200893 1526-1540. (doi:10.1210/ jc.2008-0125)

2 Boscaro M \& Arnaldi G. Approach to the patient with possible Cushing's syndrome. Journal of Clinical Endocrinology and Metabolism 200994 3121-3131. (doi:10.1210/jc.2009-0612)

3 Chiodini I, Mascia ML, Muscarella S, Battista C, Minisola S, Arosio M, Santini SA, Guglielmi G, Carnevale V \& Scillitani A. Subclinical hypercortisolism among outpatients referred for osteoporosis. Annals of Internal Medicine 2007147 541-548. (doi:10.7326/0003-4819-147-8200710160-00006)

4 Terzolo M, Reimondo G, Chiodini I, Castello R, Giordano R, Ciccarelli E, Limone P, Crivellaro C, Martinelli I, Montini M et al. Screening of Cushing's syndrome in outpatients with type 2 diabetes: results of a prospective multicentric study in Italy. Journal of Clinical Endocrinology and Metabolism 201297 3467-3475. (doi:10.1210/jc.2012-1323)

5 Fong BM, Tam S \& Leung KS. Improved liquid chromatographytandem mass spectrometry method in clinical utility for the diagnosis of Cushing's syndrome. Analytical and Bioanalytical Chemistry 2010396 783-790. (doi:10.1007/s00216-009-3247-1)

6 Taylor RL, Machacek D \& Singh RJ. Validation of a high-throughput liquid chromatography-tandem mass spectrometry method for urinary cortisol and cortisone. Clinical Chemistry 200248 1511-1519.

7 Djedovic NK \& Rainbow SJ. Detection of synthetic glucocorticoids by liquid chromatography-tandem mass spectrometry in patients being investigated for Cushing's syndrome. Annals of Clinical Biochemistry 201148 542-549. (doi:10.1258/acb.2011.010250)

8 Cooper MS \& Stewart PM. 11 $\beta$-Hydroxysteroid dehydrogenase type 1 and its role in the hypothalamus-pituitary-adrenal axis, metabolic syndrome, and inflammation. Journal of Clinical Endocrinology and Metabolism 200994 4645-4654. (doi:10.1210/jc.2009-1412)

9 Palermo M, Shackleton CH, Mantero F \& Stewart PM. Urinary free cortisone and the assessment of $11 \beta$-hydroxysteroid dehydrogenase activity in man. Clinical Endocrinology 199645 605-611. (doi:10.1046/ j.1365-2265.1996.00853.x)

10 Ceccato F, Barbot M, Zilio M, Ferasin S, Occhi G, Daniele A, Mazzocut S, Iacobone $\mathrm{M}$, Betterle $\mathrm{C}$, Mantero $\mathrm{F}$ et al. Diagnostic performance of salivary cortisol in the diagnosis of Cushing's syndrome, adrenal incidentaloma and adrenal insufficiency. European Journal of Endocrinology 2013169 31-36. (doi:10.1530/EJE-13-0159)

11 Alberti KG, Eckel RH, Grundy SM, Zimmet PZ, Cleeman JI, Donato KA, Fruchart JC, James WP, Loria CM \& Smith SC Jr. Harmonizing the metabolic syndrome: a joint interim statement of the International Diabetes Federation Task Force on Epidemiology and Prevention; National Heart, Lung, and Blood Institute; American Heart Association; World Heart Federation; International Atherosclerosis Society; and International Association for the Study of Obesity. Circulation 2009120 1640-1645. (doi:10.1161/CIRCULATIONAHA.109.192644)

12 Barbot M, Albiger N, Koutroumpi S, Ceccato F, Frigo AC, Manara R, Fassina A, Gardiman MP, Scanarini M, Mantero F et al. Predicting late recurrence in surgically treated patients with Cushing's disease. Clinical Endocrinology 201379 394-401. (doi:10.1111/cen.12133)

13 Antonelli G, Artusi C, Marinova M, Brugnolo L, Zaninotto M, Scaroni C, Gatti R, Mantero F \& Plebani M. Cortisol and cortisone ratio in urine: LC-MS/MS method validation and preliminary clinical application. Clinical Chemistry and Laboratory Medicine 201452 213-220. (doi:10.1515/cclm-2013-0471)

14 Persichilli S, Gervasoni J, Iavarone F \& Zuppi C. A simple liquid chromatography-tandem mass spectrometry method for urinary free cortisol analysis: suitable for routine purpose. Clinical Chemistry and Laboratory Medicine 201048 1433-1437. (doi:10.1515/CCLM.2010.282)

15 Monaghan PJ, Keevil BG \& Trainer PJ. Mass spectrometry for the endocrine clinic-much to digest. Clinical Endocrinology $2013 \mathbf{7 8}$ 344-346. (doi:10.1111/cen.12125)

16 Stiefel P, García-Morillo JS, Jimenez L, Pamies E, Miranda ML, Carneado J, Villar J \& Leal-Cerro A. Role of ketoconazole treatment in urinary-free cortisol-to-cortisone and tetrahydrocortisol-to-tetrahydrocortisone ratios in nonectopic Cushing's syndrome. Endocrine 2002 18 279-284. (doi:10.1385/ENDO:18:3:279)

17 Petersenn S, Newell-Price J, Findling JW, Gu F, Maldonado M, Sen K, Salgado LR, Colao A \& Biller BM. High variability in baseline urinary free cortisol values in patients with Cushing's disease. Clinical Endocrinology 201480 261-269. (doi:10.1111/cen.12259)

18 Carrasco CA, Coste J, Guignat L, Groussin L, Dugué MA, Gaillard S, Bertagna X \& Bertherat J. Midnight salivary cortisol determination for assessing the outcome of transsphenoidal surgery in Cushing's disease. Journal of Clinical Endocrinology and Metabolism 200893 4728-4734. (doi:10.1210/jc.2008-1171)

19 Nunes ML, Vattaut S, Corcuff JB, Rault A, Loiseau H, Gatta B, Valli N, Letenneur L \& Tabarin A. Late-night salivary cortisol for diagnosis of overt and subclinical Cushing's syndrome in hospitalized and ambulatory patients. Journal of Clinical Endocrinology and Metabolism 200994 456-462. (doi:10.1210/jc.2008-1542)

20 Bou-Khalil R, Baudry C, Guignat L, Carrasco C, Guibourdenche J, Gaillard S, Bertagna X \& Bertherat J. Sequential hormonal changes in 21 patients with recurrent Cushing's disease after successful pituitary surgery. European Journal of Endocrinology 2011165 729-737. (doi:10.1530/EJE-11-0424)

21 Alexandraki KI \& Grossman AB. The ectopic ACTH syndrome. Reviews in Endocrine and Metabolic Disorders 201011 117-126. (doi:10.1007/ s11154-010-9139-z)

Received 22 January 2014

Revised version received 10 April 2014

Accepted 10 April 2014 